\title{
Análisis del mercado del Shampoo Naturenix
}

\author{
Ciro Moisés Romo Puebla ${ }^{9}$ \\ Víctor Manuel Delgado Moreno ${ }^{10}$ \\ Arturo Alejandro Meza Sánchez Laurel ${ }^{11}$
}

\section{Resumen}

El presente representa un apoyo a un grupo de emprendedores de la División de Ingeniería de la Universidad de Sonora quienes requerían apoyo para obtener información para tomar decisiones acertadas e implementar estrategias adecuadas en la comercialización de su producto: un Shampoo (Naturenix) que ayuda a evitar la pérdida de cabello y además lo fortalece, su característica principal es que está hecho con ingredientes naturales. La ardua competencia en el sector, así como el poco conocimiento del comportamiento del mercado hacen necesaria esta investigación, logrando obtener la información necesaria y aplicable en las decisiones inmediatas de la empresa.

\section{Palabras clave: investigación, posicionamiento,} AGEB.

\section{Área temática y mesa}

Ejemplo: Marketing y Consumo: Investigación de Mercados.

\section{Introducción}

Naturenix está dirigido a todas aquellas personas, tanto a hombres y mujeres que sufren de algún problema en el cuero cabelludo, que desean recuperar el cabello perdido a través del tiempo, evitar la caída, conservar un cabello fuerte, con volumen, lleno de vida y brillo, además de otros beneficios. Así como también en algunos casos de niños que tienen problemas con el crecimiento del cabello, cabello muy delgado que tarde mucho en crecer o por alguna otra enfermedad. El cuidado al momento de la aplicación es el mismo que para un Shampoo convencional, cabe mencionar que es un Shampoo tratamiento y la constancia dará los resultados esperados más rápida y efectivamente.

La idea de dar a conocer un producto que tenga como principal función el renacimiento del cabello se ha venido dando desde hace tiempo.

Esto data desde 1995 en que el señor hoy finado Manuel Tabardillo Castro descubrió la combinación perfecta de hierbas naturales para la elaboración de un producto, en este caso Shampoo, que tiene la función de evitar la caída y estimular el crecimiento del cabello, este descubrimiento se dio gracias a la gran amistad que tenía don Manuel con gente perteneciente a la tribu yaqui, los cuales le platicaron que hierbas eran buenas para tratar el cabello y que al unirlas se podía obtener los sorprendentes resultados antes mencionados.

Esta fórmula, al tener sus principales orígenes de una tribu que ha subsistido durante siglos y de la más representativas del estado de sonora, nos da una gran

\footnotetext{
${ }^{9}$ Universidad de Sonora. Departamento de Contabilidad. Correo Electrónico: cromo@eca.uson.mx Tel. 2592211

${ }^{10}$ Universidad de Sonora. Departamento de Contabilidad. Correo Electrónico: vdelgado@eca.uson.mx Tel. 2592211

${ }^{11}$ Universidad de Sonora. Departamento de Contabilidad. Correo Electrónico:ameza@eca.uson.mx Tel. 2592211
} 
confianza en la efectividad del producto ya que realmente los grandes conocedores de la herbolaria mexicana, han pertenecido a tribus indígenas, las cuales en la mayoría de sus enfermedades encuentran cura en recetas naturales.

El señor ya finado Manuel Tabardillo Castro transmitió a su vez el conocimiento de dicha fórmula a su nieto Joel Tabardillo Rojas quien después de terminar sus estudios de nivel superior, se asocia con 3 amigos egresados de su misma carrera (Ing. industrial y de Sistemas) e inician lo que hoy es la empresa Compañía

JAYAN.

Compañía Jayan se encarga de fabricar y distribuir este producto y al cual se le dio por nombre Shampoo "Fénix Natural" haciendo alusión al ave de la mitología griega que resurgía de las cenizas y que nunca moría, así como también a la naturaleza por el origen de sus ingredientes.

Después se tomó una decisión muy importante que el producto cambiara su nombre a "Naturenix"

Durante el desarrollo que ha tenido este producto para llevarse a la comercialización, ha pasado primeramente por la aprobación de expertos asesores, diversas pruebas hechas con personas jóvenes y mayores con edades de 18 a 60 años, dándonos un muy buen resultado.

\section{Misión}

"Fabricar productos naturales con tecnología de vanguardia, sin dañar el medio ambiente, logrando la completa satisfacción de nuestros clientes y socios, llevando a cabo una buena relación con nuestros proveedores y contribuir con nuestra sociedad"

\section{Visión}

Lograr un $25 \%$ de aceptación dentro del mercado de productos naturales, extender nuestra línea un 50\%, reflejar nuestra calidad en cada uno de nuestros productos y así alcanzar la confianza y satisfacción total de nuestros consumidores al ser así la mejor opción para la solución de sus problemas con la caída y regeneración de cabello.
25\% de aceptación dentro del mercado de productos naturales.

Extender nuestra línea un 10\%, agregando un acondicionador.

Reflejar $100 \%$ nuestra calidad en cada uno de nuestros productos.

\section{Marco teórico}

Las organizaciones que se dirigen a un mercado no expandible deben aceptar el tamaño del mercado y concentrar sus esfuerzos en conseguir una mayor participación de mercado para su producto (el nivel de demanda selectiva del producto de la empresa).

Las empresas también deben comparar su participación de mercado con su participación de mercado potencial. El resultado de esta comparación se denomina índice de penetración de la empresa. Si éste es bajo, significa que la empresa puede aumentar su participación de mercado considerablemente (Kotler, 2007, p. 128).

Los factores subyacentes que la limitan podrían ser:

\section{Poca relevancia de marca \\ Poca disponibilidad de marca \\ Beneficios deficientes \\ Precio demasiado elevado}

"Las empresas deben calcular cómo podrían incrementar su participación de mercado invirtiendo en eliminar los posibles errores para ver qué inversiones generarían la mayor mejora en el índice de penetración" (Best, 2007)

La función de demanda del mercado no es una imagen de la demanda en el tiempo, sino que lo que muestra la curva, más bien, son diferentes combinaciones entre los pronósticos de demanda asociados con posibles gastos del sector en actividades de marketing, durante un periodo determinado. 
En un momento dado sólo puede existir un nivel de gasto en actividades de marketing dentro de cada sector. La demanda de mercado correspondiente a este nivel se denomina estimación de mercado.

La estimación de mercado muestra la demanda de mercado prevista, no la demanda máxima de mercado. Para estimar esta última se debe visualizar el nivel de demanda de mercado resultante de un nivel de gasto sectorial de marketing "muy elevado", a partir del cual los sucesivos aumentos en el gasto apenas surten efectos en la demanda. El potencial de mercado es el límite al cual se aproxima la demanda del mercado cuando los gastos de marketing del sector tienden a infinito, en un determinado entorno de marketing.

\section{La Investigación de Mercado}

Las pequeñas y medianas empresas (PYMES) saben que el mercado es cada vez más difícil y sienten que el consumidor es cada vez más exigente, los competidores aumentan, entran marcas internacionales.

La investigación de mercados en cualquier clase de empresa no es para explorar lo que ya se sabe y se conoce (Díaz, 2015). Cada segundo que transcurre en el mundo empresarial se toman decisiones de negocios, pero siendo el ser humano un individuo de costumbres, las empresas adoptan dichas costumbres, a final de cuentas son manejadas por seres humanos. Dichas costumbres se vuelven obsoletas ante un mercado cambiante y en constante evolución, lo cual obliga a las empresas a evolucionar también y buscar información sobre el mercado.

La investigación de mercados es un medio clave para conocer el entorno. El conocimiento del entorno ayuda a una empresa no solo a alterar su mezcla de marketing presente, sino también a aprovechar nuevas oportunidades. Un segundo rol de la investigación es la función diagnóstica, en la que se explican datos y/o acciones. El último rol de la investigación es la función predictiva.
El fin de un estudio exploratorio ocurre cuando los investigadores de mercados están convencidos de que han descubierto las principales dimensiones del problema. Pueden haber definido una serie de preguntas que pueden usarse como guías específicas para un detallado diseño de investigación. O pueden haber desarrollado varias ideas potenciales sobre posibles causas de un problema específico de importancia para la gerencia (McDaniel, 2016, o. 16).

También pueden haber determinado que otros factores son posibilidades tan remotas que pueden ignorarse sin riesgo alguno en cualquier estudio posterior. Finalmente, los investigadores pueden terminar la exploración porque creen que no es necesario investigar más o que no es posible hacerlo en ese momento debido a restricciones de tiempo, dinero u otras.

\section{Análisis situacional (FODA)}

El análisis FODA es una herramienta que nos permite conformar un cuadro de la situación actual de la empresa u organización, permitiendo de esta manera obtener un diagnóstico preciso que permita en función de ello tomar decisiones acordes con los objetivos y políticas formulados. (Ver Texto 1)

\section{Conocimiento del mercado}

\section{Importancia de la segmentación}

La segmentación de mercado es una forma de buscar nuevas oportunidades en el mercado total a través del conocimiento real de los consumidores. Se lleva a cabo a través de un proceso que consta de 3 etapas: Estudio, Análisis y Preparación de perfiles. El segmento de mercado debe de ser homogéneo a su interior, heterogéneo al exterior, con un número suficiente de consumidores para que sea rentable; $y$ operacional, es decir, que incluya dimensiones demográficas para poder trabajar adecuadamente en la plaza y promoción del producto. Los segmentos van cambiando por ello es importante realizar la segmentación de forma periódica. (Ver Texto 2) 
Texto 1

\begin{tabular}{|c|c|c|}
\hline & FORTALEZAS & DEBILIDADES \\
\hline COSTO DE PRODUCCION & $\begin{array}{l}\text { - Desplazamiento rápido de } \\
\text { lotes de producción al } \\
\text { mercado. } \\
\text { - Se consigue materia prima } \\
\text { barata. }\end{array}$ & $\begin{array}{l}\text { - Métodos rudimentarios para la } \\
\text { producción. } \\
\text { - Altos costos porque se produce lo } \\
\text { necesario. } \\
\text { - Materia prima flotante ya que la } \\
\text { situación climatologia podria afectar. }\end{array}$ \\
\hline $\begin{array}{l}\text { CAPACIDADES DE } \\
\text { COMERCIALIZACION }\end{array}$ & $\begin{array}{l}\text { - Distribuidores fieles. } \\
\text { - Trato directo con los } \\
\text { proveedores del producto. } \\
\text { - Buenas relaciones con los } \\
\text { vendedores en los puntos } \\
\text { de venta. } \\
\text { - Apoyo publicitario por las } \\
\text { cadenas comerciales. }\end{array}$ & $\begin{array}{l}\text { - Limitación para cubrir el mercado } \\
\text { demandante. } \\
\text { - Escasos canales de distribución. } \\
\text { - Poca publicidad en medios masivos de } \\
\text { comunicación. } \\
\text {-Altos costos de envios de transporte al } \\
\text { punto de venta. }\end{array}$ \\
\hline $\begin{array}{l}\text { CAPACIDAD DE LOS } \\
\text { EMPLEADOS }\end{array}$ & $\begin{array}{l}\text { - Jóvenes comprometidos } \\
\text { con visión. } \\
\text { - Buenas relaciones entre } \\
\text { los accionistas. } \\
\text { - Ambiente de trabajo } \\
\text { relajado y efectivo. }\end{array}$ & $\begin{array}{l}\text { - Falta de poder de negociación. } \\
\text { - Falta de experiencia. } \\
\text { - Falta de asignación } \\
\text { responsabilidades. }\end{array}$ \\
\hline
\end{tabular}

\begin{tabular}{|c|c|c|}
\hline & AMENAZAS & OPORTUNIDADES \\
\hline SOCIAL & $\begin{array}{l}\text { - El mercado se resista al } \\
\text { cambio. } \\
\text { - Conflictos con los distribuidores } \\
\text { de los productos. } \\
\text { - Que la gente se incline por los } \\
\text { productos más comerciales. }\end{array}$ & $\begin{array}{l}\text { - Posicionamiento en la mente de los } \\
\text { consumidores aquí se aplica la } \\
\text { comunicación de boca en boca. } \\
\text { - Fidelidad de los consumidores hacia el } \\
\text { producto por los beneficios percibidos. }\end{array}$ \\
\hline DEMOGRAFICO & $\begin{array}{l}\text { - La distancia entre el proveedor } \\
\text { y la planta. } \\
\text { - Obstáculos e imprevistos al } \\
\text { posesionarte en otro lugar. } \\
\text { - Crisis económica e inflación. }\end{array}$ & $\begin{array}{l}\text { - Es un producto regional. } \\
\text { - Fácil acceso a los lugares a llegar por } \\
\text { la cercania. } \\
\text { - No hay muchos competidores en la } \\
\text { región. } \\
\text { - Hay mucho mercado para abarcar. }\end{array}$ \\
\hline ECONOMIAS & $\begin{array}{l}\text { Impuestos agregados al producto. } \\
\text { Devaluación. } \\
\text { Aumento en los fletes y costos de } \\
\text { la materia prima. }\end{array}$ & $\begin{array}{l}\text { Pocos competidores en la región para } \\
\text { llegar a nuevas mercados teniendo una } \\
\text { disminución en costo. } \\
\text { Poder adquisitivo alto. }\end{array}$ \\
\hline
\end{tabular}




\begin{tabular}{|c|c|c|}
\hline & FORTALEZAS & DEBILIDADES \\
\hline $\begin{array}{l}\text { RECURSOS } \\
\text { FINANCIEROS }\end{array}$ & $\begin{array}{l}\text { - Apoyo gubernamental. } \\
\text { - No tienen gastos fijos. } \\
\text { - Fluidez de capital para ser } \\
\text { invertido en otras cosas. } \\
\text { - Fácil acceso al crédito y apoyo } \\
\text { de programas } \\
\text { gubernamentales a jóvenes. }\end{array}$ & $\begin{array}{l}\text { - Falta de contador, hace tener gastos } \\
\text { innecesarios no planeados, o mala } \\
\text { organización de los recursos obtenidos. } \\
\text { - No tienen presupuesto asignado para } \\
\text { el departamento de mercado. }\end{array}$ \\
\hline $\begin{array}{l}\text { TECNOLOGIA } \\
\text { DISPONIBLE }\end{array}$ & $\begin{array}{l}\text { - Su fabricación no es muy } \\
\text { complicada. } \\
\text { - Facial acceso a nuevas } \\
\text { maquinarias, con préstamos } \\
\text { del gobierno. }\end{array}$ & $\begin{array}{l}\text { - No cuentan con tecnología de } \\
\text { vanguardia. } \\
\text { - Poco presupuesto para la renovación } \\
\text { de la planta de producción. }\end{array}$ \\
\hline $\begin{array}{l}\text { IMAGEN DE LA } \\
\text { COMPANNIA O DE LA } \\
\text { MARCA }\end{array}$ & $\begin{array}{l}\text { - Confiable al verse como un } \\
\text { producto natural. } \\
\text { - Consumidores son fieles } \\
\text { gracias a su imagen. } \\
\text { - Seguridad al ver su } \\
\text { consistencia que indica ser un } \\
\text { producto natural. }\end{array}$ & $\begin{array}{l}\text { - Una imagen poco llamativa y atractiva, } \\
\text { muy tradicional. } \\
\text { - Pasa desapercibida. } \\
\text { - La identidad del producto no es } \\
\text { amigable con el posible consumidor. }\end{array}$ \\
\hline
\end{tabular}

\begin{tabular}{|c|c|c|}
\hline & AMENAZAS & OPORTUNIDADES \\
\hline TECNOLOGICA & $\begin{array}{l}\text { - Que avance mucho la } \\
\text { tecnologia y se quede la } \\
\text { maquinaria de la empresa } \\
\text { obsoleta. } \\
\text { Que los cogidos de barra sean } \\
\text { diferentes en paises. } \\
\text { - Insuficiente capacidad del } \\
\text { personal para el manejo de la } \\
\text { tecnologia. }\end{array}$ & $\begin{array}{l}\text { - Adquisición de tecnologias nuevas } \\
\text { para la planta de producción. } \\
\text { - Actualización y capacitación constante } \\
\text { de manejo e implementación de la } \\
\text { nueva tecnologia para el proceso } \\
\text { productivo. }\end{array}$ \\
\hline $\begin{array}{l}\text { POLITICAS / } \\
\text { LEGALES }\end{array}$ & $\begin{array}{l}\text { - Los requisitos que se pidan al } \\
\text { momento de exportar a otro } \\
\text { pais que no se pueden cumplir. } \\
\text { - Impuestos muy elevados. } \\
\text { - Aranceles muy altos al } \\
\text { exportar. }\end{array}$ & $\begin{array}{l}\text { - No tienen limitantes en el país a } \\
\text { exportar para que entre el producto. } \\
\text { - Tratados de México con otros paises. } \\
\text { - Respaldo del gobierno. }\end{array}$ \\
\hline $\begin{array}{l}\text { FUERZAS } \\
\text { COMPETITIVAS }\end{array}$ & $\begin{array}{l}\text { - La competencia disminuya el } \\
\text { precio y gane mercado. } \\
\text { - Recursos financieros altos. } \\
\text { - Inversión a mercados } \\
\text { atascados por la competencia } \\
\text { al mercado de Fénix. } \\
\text { - Llegada de un competidor } \\
\text { potencial no esperado. }\end{array}$ & $\begin{array}{l}\text { - El producto tiene una formula única. } \\
\text { - Se posiciona en tiendas conocidas por } \\
\text { los distribuidores. } \\
\text { - Agregar técnicas de investigación de } \\
\text { mercados como mistery shopper y } \\
\text { benchmarking. } \\
\text { - Posicionarse en mercados } \\
\text { desatendidos por la competencia } \\
\text { apoyándose de la publicación semanal } \\
\text { en periódicos de ofertas de los } \\
\text { distribuidores que cuenten con ellas. }\end{array}$ \\
\hline
\end{tabular}




\section{Texto 2}

Ejercicio de segmentación

\begin{tabular}{|c|c|}
\hline $\begin{array}{l}\text { Fieles } \\
\text { - } \\
\text { - } \text { Personas entre } 25-45 \text { años. } \\
\text { shampoo si lo conocen. } \\
\text { - No ahorran la mayoria de su sueldo. } \\
\text { - Su forma de vestir es informal. } \\
\text { - Su caracter es extrovertido y sociable. } \\
\text { - Esperan la quincena para hacer sus } \\
\text { gastos personales. } \\
\text { - No se preocupan mucho por su } \\
\text { presentación personal. } \\
\text { - Les gustan las peliculas de humor y } \\
\text { acción. }\end{array}$ & $\begin{array}{l}\text { Ocultos } \\
\text { - Personas de } 30-45 \text { años. Las esposa o madres } \\
\text { compran el producto por ellos A los hombres no les } \\
\text { gusta adquirir el shampoo y que la gente los vea. } \\
\text { - Adquieren el producto a cualquier precio. } \\
\text { - Les averguenza que sepan que se les esta } \\
\text { cayendo el pelo. } \\
\text { - Su carácter es por lo general sociable. } \\
\text { - Se preocupan por su apariencia fisica. } \\
\text { - Lestinan su gasto paseos y diversión. } \\
\text { - Participan en actividades altruistas. }\end{array}$ \\
\hline $\begin{array}{l}\text { Los high } \\
\text { - Son personas que tienen un rango de } \\
\text { edad de } 23-30 \text { años. } \\
\text { - Son aquellas personas que conocen el } \\
\text { producto y lo adquieren en tiendas que } \\
\text { están de acuerdo con su status. } \\
\text { - Conocieron el producto por medio de un } \\
\text { rumor y sus conocidos } \\
\text { - Destinan su dinero en el gasto personal } \\
\text { - y belleza, viajes y diversión } \\
\text { - Personas que sufren caída del cabello } \\
\text { - Van a gimnasios se preocupa por su } \\
\text { - Sisico } \\
\text { - Non personas sociables y extrovertido } \\
\text { - Gustan de peliculas de humor } \\
\text { - Normalmente visten de manera casual } \\
\text { - Son aquellas que realizan actividades } \\
\text { extras }\end{array}$ & $\begin{array}{l}\text { Precavidos } \\
\text { - Edad de } 30-45 \text { años } \\
\text { - Previenen la caida del pelo } \\
\text { - } \text { Cedican parte de su dinero al cuidado personal } \\
\text { - Aceptan que puede tener problemas de calvicie } \\
\text { - No se arriesgan con otros productos } \\
\text { - No pagan mas de lo que en realidad cuesta } \\
\text { - Algunas de estas personas ya utilizan este } \\
\text { - Sroducto } \\
\text { - Len personas introvertidas } \\
\text { - No son personas Sociables }\end{array}$ \\
\hline
\end{tabular}


Tabla de segmentación con todas las variables

\begin{tabular}{|c|c|}
\hline Edad & 18 años en adelante \\
\hline Sexo & Hombres - Mujeres \\
\hline Estado civil & Indiferente \\
\hline Religión & Indiferente \\
\hline $\begin{array}{c}\text { Nivel } \\
\text { Socioeconómico }\end{array}$ & $A / B, C^{+}$y $C$ \\
\hline Nivel de instrucción & No necesario \\
\hline $\begin{array}{l}\text { Características de } \\
\text { vivienda }\end{array}$ & Vivienda con todos los servicios \\
\hline Unidad geográfica & Mercado Regional (Sonora) \\
\hline $\begin{array}{l}\text { Condiciones } \\
\text { Geográficas }\end{array}$ & $\begin{array}{l}\text { Clima Húmedo con lluvias en verano, ecosistema desértico, } \\
\text { ciudades con ecosistemas muy calurosos }\end{array}$ \\
\hline Raza & Indistinta \\
\hline Tipo de población & Ciudades-Metrópolis \\
\hline Grupos de referencia & $\begin{array}{l}\text { Las personas que dicen que es importante el uso, así como la } \\
\text { familia, los amigos. }\end{array}$ \\
\hline Clase social & Media y Alta \\
\hline Personalidad & $\begin{array}{l}\text { Fieles, Ocultos, los high y los precavidos (Véase la } \\
\text { descripción de cada uno en el cuadro al final de esta tabla) }\end{array}$ \\
\hline Cuitura & Media \\
\hline Ciclo de vida familiar & $\begin{array}{l}\text { Mujeres jóvenes, casadas con hijos en el hogar, casada sin } \\
\text { hijos, solteras mayores }\end{array}$ \\
\hline Motivos de compra & Necesidad \\
\hline Frecuencia de uso & Tres veces por semana \\
\hline Ocasión de uso & Frecuente \\
\hline Tasa de uso & Mediana \\
\hline Lealtad & Mucha lealtad \\
\hline $\begin{array}{l}\text { Disposición de } \\
\text { compra }\end{array}$ & Tiene que haber disposición \\
\hline
\end{tabular}

\section{Texto 3}

\section{Farmacia Benavides}

Cre-C lo venden en una cajita con 3 botellitas de $250 \mathrm{ml} \mathrm{c/u}$, a $\$ 299$ la cajita. Que si se vende muy seguido. Si conocen Naturenix, pero que no lo venden, y muchas veces han llegado a preguntar por él. Si saben dónde se vende, saben que lo venden en farmacias Kino, y en farmacias Santa Fe.

\section{Costco}

Cre-C lo vende en una caja con dos botellas de $500 \mathrm{ml}$, es un Shampoo y un acondicionador, al precio de $\$ 189$ por caja con las 2 presentaciones. No venden Shampoo Naturenix natural, y no supieron acerca de él. 
Texto 4

\begin{tabular}{|l|c|c|}
\hline \multicolumn{1}{|c|}{ Atributo del producto } & Naturenix & Competencia \\
\hline Fórmula original & $\mathrm{X}$ & \\
\hline Concentrado de la formula en shampoo & $\mathrm{X}$ & \\
\hline Recomendación de boca en boca & $\mathrm{X}$ & \\
\hline Resultados rápidos & $\mathrm{X}$ & \\
\hline Beneficios en el crecimiento de cabello & $\mathrm{X}$ & \\
\hline Garantía & & $\mathrm{X}$ \\
\hline Calidad del producto & $\mathrm{X}$ & \\
\hline Diseño & & $\mathrm{X}$ \\
\hline Empaque & & $\mathrm{X}$ \\
\hline
\end{tabular}

\section{Benchmarking}

Benchmarking es el proceso continuado de medición de los productos, servicios y prácticas propias en comparación con las de los más duros competidores o con las de aquellas compañías líderes en su sector.

Para una empresa el objetivo del benchmarking desde el punto de vista comercial es comparar su propio producto con los de sus principales competidores, tomando para cada atributo clave del producto el líder o mejor situado en cada caso, y así poder establecer un resultado o valoración global comparativa. (Ver Texto 3 y 4)

\section{Selección de los atributos relevantes del} producto.

¿Cuáles son los atributos más relevantes para la categoría de producto?
Hallados a través de:

Estudio cualitativo/cuantitativo 0 muestro seleccionado de consumidores.

Si no es posible, estimados o partir de lo experiencia.

Los atributos del shampoo Naturenix son, su original formula, consistencia del concentrado al fondo del shampoo, es recomendado de boca en boca lo cual lo hace más confiable, los buenos resultados y beneficios que genera.

2. Valorar qué marco del mercado es superior para cada atributo.

Para cada atributo del producto, ¿cuál es la mejor marca valorada del mercado? (Ver Texto 5)

Texto 5

\begin{tabular}{|l|c|}
\hline \multicolumn{1}{|c|}{ Atributo del producto } & Mejor marca valorada \\
\hline Formula original & Cre-C \\
\hline Concentrado de la formula en shampoo & Cre-C \\
\hline Recomendación de boca en boca & Naturenix \\
\hline Resultados rápidos & Naturenix \\
\hline Beneficios en el crecimiento de cabello & Naturenix \\
\hline Garantia & Cre-C \\
\hline Calidad del producto & Cre-C \\
\hline Diseño & Cre-C \\
\hline Empaque & Cre-C \\
\hline
\end{tabular}




\section{ESTRATEGIAS DEL PRODUCTO}

\section{Balanza comercial}

La situación contable o financiera de una empresa puede ser aceptable en un momento dado, pero en pocos meses puede cambiar radicalmente si sus verdaderos activos los productos están sucumbiendo ante una competencia que lo está haciendo mejor o si la gama de productos propios se está quedando obsoleta.

\section{Balance comercial (I)}

\begin{tabular}{|c|c|c|c|}
\hline \multirow{12}{*}{ 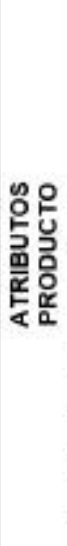 } & $\begin{array}{l}\text { Valoración: } \\
0,1,2,3,4,5,6,7,8,9,10\end{array}$ & $\begin{array}{c}\text { Activo } \\
\text { (nuestro } \\
\text { producto) } \\
\text { Naturenix }\end{array}$ & $\begin{array}{c}\text { Pasivo } \\
\text { (principal } \\
\text { competidor) } \\
\text { Cre-ce }\end{array}$ \\
\hline & 1. Fórmula-núcleo-materia & 10 & 8 \\
\hline & 2. Calidad & 9 & 9 \\
\hline & 3. Diseño & 5 & 7 \\
\hline & 4. Surtido gama & 0 & 5 \\
\hline & 5. Precio & 9 & 6 \\
\hline & 6. Envase & 7 & 5 \\
\hline & 7. Marca & 5 & 10 \\
\hline & 8. Servicio & 8 & 7 \\
\hline & 9. Imagen producto & 4 & 6 \\
\hline & 10. Imagen empresa & 6 & 5 \\
\hline & Total & 63 & 68 \\
\hline
\end{tabular}

\section{Matriz General Electric}

Este sistema de análisis fue desarrollado por el departamento de planificación de la compañía General Electric, y realmente aporta una información útil en el diagnóstico del producto. Consiste en situar a los productos de la empresa en un plano con dos coordenadas: en ordenadas, el atractivo o la atracción que tiene el mercado para la empresa; en abscisas, la posición que tiene la empresa en dicho mercado.

Este tipo de análisis complementa el análisis del portafolio. Sirve para afinarlo y poder tomar decisiones más seguras. En definitiva, aporta un diagnóstico más claro de la situación con lo que la planificación estratégica del producto puede estar mejor orientada y ser más segura.
El balance comercial tiene la misión de solventar estos problemas y poder medir, desde una óptica eminentemente comercial, cuál es el valor de la compañía a través de sus productos.

EI balance comercial tiene dos partes bien diferenciadas: Una basada en los atributos del producto, otra basada en los atributos marketing.

\begin{tabular}{|c|c|c|c|c|}
\hline & Valoración & Ponderación & Ponderación & (Indiferencia) \\
\hline \multicolumn{2}{|c|}{ De sólo 10 puntos a 1 de las 3 casillas } & $\begin{array}{l}\text { (Nuestro } \\
\text { producto) } \\
\text { Naturenix }\end{array}$ & $\begin{array}{c}\text { (Principal } \\
\text { competidor) } \\
\text { Cre-ce }\end{array}$ & \\
\hline 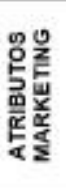 & $\begin{array}{l}\text { A. Aportación al metcado } \\
\text { B. Fideidad } \\
\text { C. Ventaja diferencial } \\
\text { D. Alta frecuencia de compra } \\
\text { E. Participacion mercado }\end{array}$ & 10 & $\begin{array}{l}10 \\
10\end{array}$ & \\
\hline & Total puntos & 20 & 30 & 0 \\
\hline & Balance & $\begin{array}{c}\text { Activo } \\
1260\end{array}$ & $\begin{array}{c}\text { Pasivo } \\
2040\end{array}$ & -780 \\
\hline
\end{tabular}

Para empresa Jayan es importante atraer a la clase media alta para el consumo de sus productos al igual que la posición de la organización se encuentra ubicada en el área media. Esto por el número de empleados con el que la empresa cuenta.

El área de rayas representa la falta de consumidores que tienen el problema y no están atacados, se podría decir que esta zona se encuentra como el mercado desatendido por nuestro producto.

La zona blanca es la interrogante de la empresa, se podría llamar la zona de espera, es la zona donde se pretende crecer y posicionar la empresa. (Ver Texto 6) 


\section{Texto 6}

\begin{tabular}{|c|c|c|c|c|c|}
\hline \multirow{2}{*}{$\begin{array}{l}\text { Atracción } \\
\text { Mercado }\end{array}$} & \multirow{2}{*}{ Ponderación } & \multicolumn{2}{|c|}{ Acondicionador } & \multicolumn{2}{|c|}{ Shampoo } \\
\hline & & Nota & Ponderación & Nota & Ponderación \\
\hline Tamafio & 15 & 20 & 1000 & 10 & 200 \\
\hline Crecimiento & 35 & 100 & 200 & 100 & 1500 \\
\hline $\begin{array}{l}\text { Estructura } \\
\text { competitiva }\end{array}$ & 10 & 10 & 500 & 50 & 100 \\
\hline Rentabilidad & 20 & 20 & 150 & 80 & 1000 \\
\hline $\begin{array}{l}\text { Medio } \\
\text { ambiente }\end{array}$ & 10 & 10 & 40 & 10 & 100 \\
\hline Legal & 5 & 10 & 300 & 10 & 50 \\
\hline Humano & 2 & 10 & 20 & 10 & 20 \\
\hline Técnico & 3 & 20 & 200 & 20 & 30 \\
\hline $\begin{array}{l}\text { Posición } \\
\text { empresa }\end{array}$ & 100 & & 1,690 & & 3,000 \\
\hline $\begin{array}{l}\text { Volumen } \\
\text { ventas }\end{array}$ & 25 & 70 & 200 & 80 & 1500 \\
\hline Crecimiento & 20 & 50 & 50 & 70 & 100 \\
\hline Participación & 10 & 100 & 700 & 20 & 400 \\
\hline $\begin{array}{l}\text { Posición } \\
\text { corporativa }\end{array}$ & 5 & 10 & 10 & 10 & 100 \\
\hline Márgenes & 30 & 100 & 1000 & 60 & 400 \\
\hline $\begin{array}{l}\text { Posición } \\
\text { tecnologica }\end{array}$ & 3 & 20 & 250 & 10 & 70 \\
\hline $\begin{array}{l}\text { Debilidades - } \\
\text { fortalezas }\end{array}$ & 2 & 10 & 100 & 10 & 50 \\
\hline Imagen & $\stackrel{5}{100}$ & 50 & $\begin{array}{c}10 \\
2,320\end{array}$ & 20 & $\begin{array}{c}200 \\
2,820\end{array}$ \\
\hline
\end{tabular}

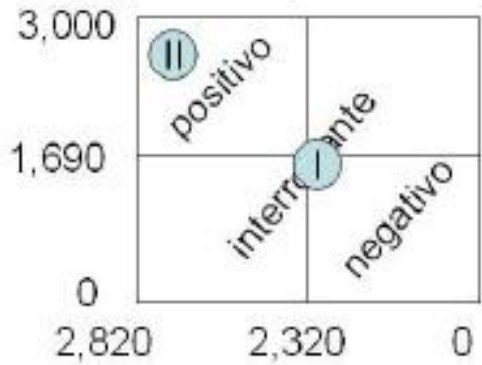

\section{Se puede observar que:}

El producto II es el precursor para el lanzamiento del producto I (acondicionador) con el producto II se impulsará el producto I al mercado. El producto II se muestra estable y con crecimiento.

El producto I con las perspectivas (shampoo) más esperadas, tiene una gran esperanza de vida en el mercado, al igual de su aceptación en el mercado.

\section{Investigación de mercado}

\section{Metodología}

Para la realización de encuestas se llevaron a cabo las siguientes actividades:

Se elaboró un cuestionario (instrumento de medición) para obtener la información necesaria.

Se calculó el tamaño de muestra (384 encuestas).

Se dividieron las encuestas en 30 Áreas geoestadísticas básicas (AGEB) elegidas al azar en la Ciudad de Hermosillo, Sonora.
Dentro de los AGEB se eligieron los hogares a visitar.

Se aplicó la encuesta a personas mayores de edad que estuvieran en el momento de la visita en el hogar y mostraran disposición a participar.

\section{Objetivo General}

El objetivo general de esta investigación de mercados, tiene como finalidad procesar la información generada desde el punto de vista de los consumidores y del producto en cuestión, en este caso el Shampoo Naturenix.

Servir de elemento de análisis en el proceso de planeación de cualquier empresa y cumplir su función como instrumento de dirección para ayudar a marcar los caminos a seguir en el desarrollo de la empresa con base en lo que desean, esperan y necesitan los consumidores.

\section{Objetivo Específico}

Detectar las necesidades del mercado desatendido, tomando en cuenta los hábitos y forma de consumo, buscando específicamente los puntos que deberán ser 
atacados a partir de los resultados que arroje la investigación que los consumidores actuales o potenciales de este tipo de productos naturales.

\section{Planteamiento de Hipótesis}

Al $80 \%$ de las personas encuestadas han utilizado en alguna ocasión un Shampoo para combatir la caída del cabello.

El $40 \%$ de los encuestados conoce el Shampoo Naturenix.

El $90 \%$ de los encuestados considera la calidad del Shampoo Naturenix como Buena.

\section{Determinación de la Muestra}

Al momento de determinar el universo, se utilizó la fórmula del universo infinito que arrojo el tamaño de la muestra de 384 encuestas, con un nivel de confianza de $95 \%$ y un margen de error del $5 \%$.

$$
n=\frac{\sigma^{2} p q}{e^{2}}=n=\frac{(1.96)^{2}(0.50)(0.50)}{(0.05)^{2}}=384
$$

Donde:

$Z=$ Confiabilidad (en este caso del 95\% equivalente a 1.96 desviaciones estándar de la media

$p=$ La probabilidad de que un evento ocurra (respuesta favorable)

$q=$ La probabilidad de que un evento no ocurra (respuesta desfavorable) $01-p$.

$e=$ Error de estimación (del 5\%)

\section{Resultados de la investigación}

Del total de las encuestas, el $67 \%$ correspondió al sexo femenino. Esto se debe a dos razones principales: (1) las encuestas fueron en casa habitación (hogares) y (2) las mujeres mostraron mayor disposición al momento de contestar la encuesta.

Las edades se concentraron en los rangos de 23 hasta los 42 años.
Sexo
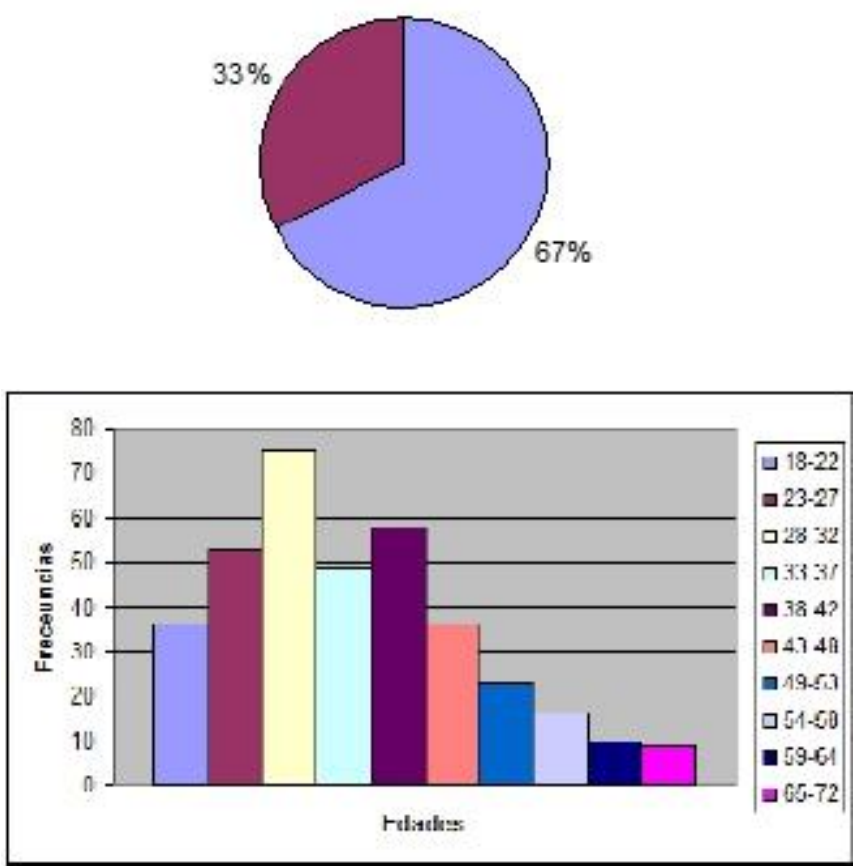

El $69 \%$ de los encuestados admitieron el estar usando o que alguna vez utilizaron algún tipo de Shampoo para la caída del cabello. El 91\% de los encuestados tenían conocimiento del producto, un 9\% no conocía en absoluto el producto.

\section{1. ¿Ha utilizado alguna vez Shampoo para prevenir la caída del cabello?}

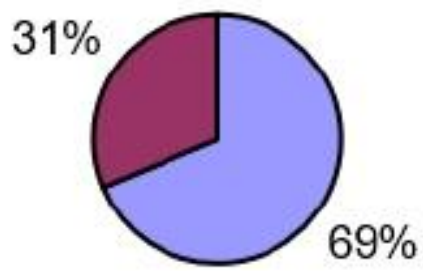




\section{2. ¿Conoce el Shampoo Naturenix?}

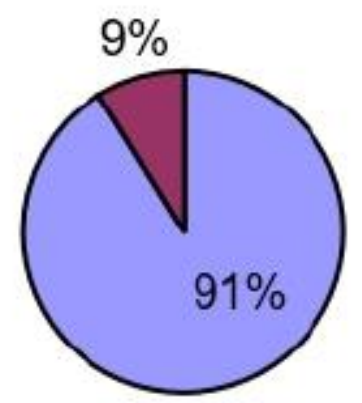

La mayoría de las personas entrevistadas consideraron que la calidad del producto es buena. Además, El producto en sí, tiene una buena imagen ante las personas encuestadas.

\section{3. ¿Cómo considera la calidad del producto?}

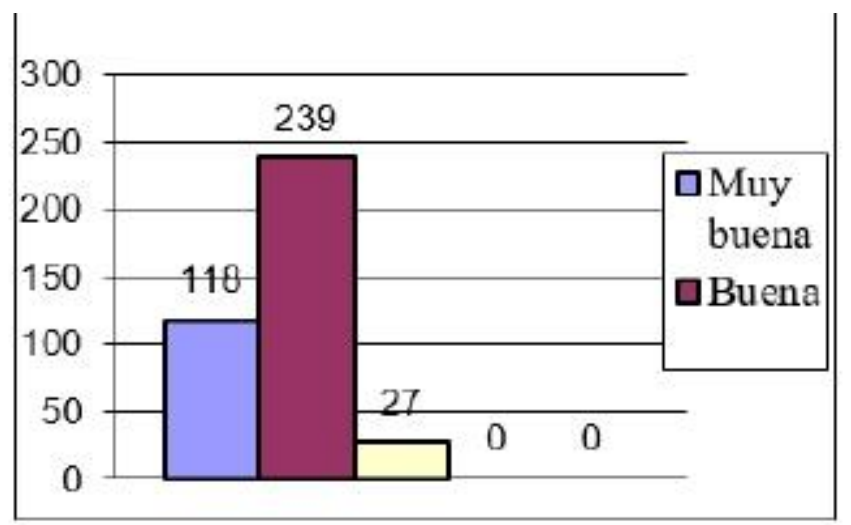

\section{4. ¿Considera la imagen del Shampoo Naturenix?}

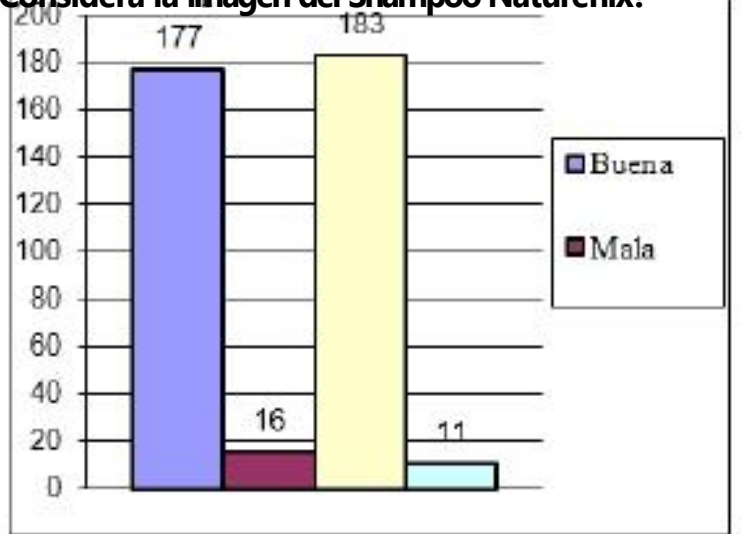

Los encuestados consideraron que el Shampoo necesita el cambio de envase como primera instancia seguido de la etiqueta. El $57 \%$ de los encuestados prefieren el nombre Fénix Natural (el actual es Naturenix), esto tomando en cuenta que la muestra se encuentra identificada con la marca, mientras que un $42 \%$ de los encuestados mostraron interés por el nombre Renassence.

\section{5. ¿Qué cambio le haría a la imagen del Shampoo?}

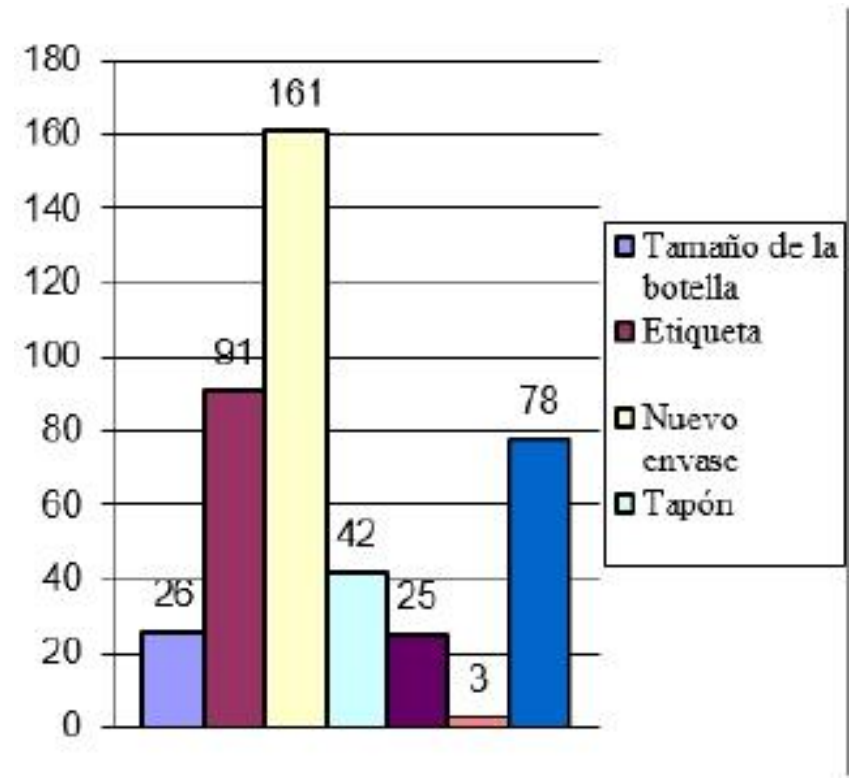

6. ¿Qué nombre le parece más adecuado?

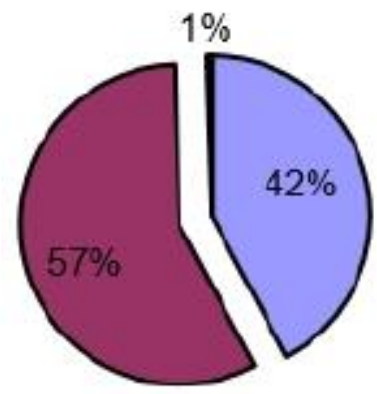


Según los encuetados, el principal distribuidor del producto es la Cadena de Farmacias Kino, seguido de Comercial Zazueta. Las farmacias Santa Fe ya no están en el mercado pero al parecer un porcentaje considerable de los encuestados las relacionan con la Marca Naturenix.

Sobre la pregunta de la posibilidad de agregar otro producto a la línea Naturenix, el acondicionador fue el producto más mencionado, seguido del jabón y el rímel También se consideró el Spray y Crema para Peinar.

\section{7. ¿En Qué lugar suele comprar Shampoo?}

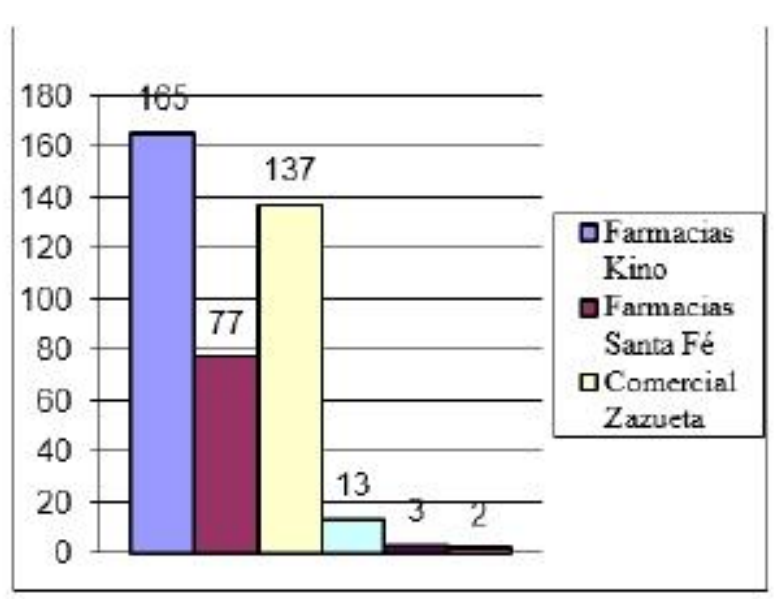

8. ¿Considera necesario agregar más productos a la línea Naturenix?

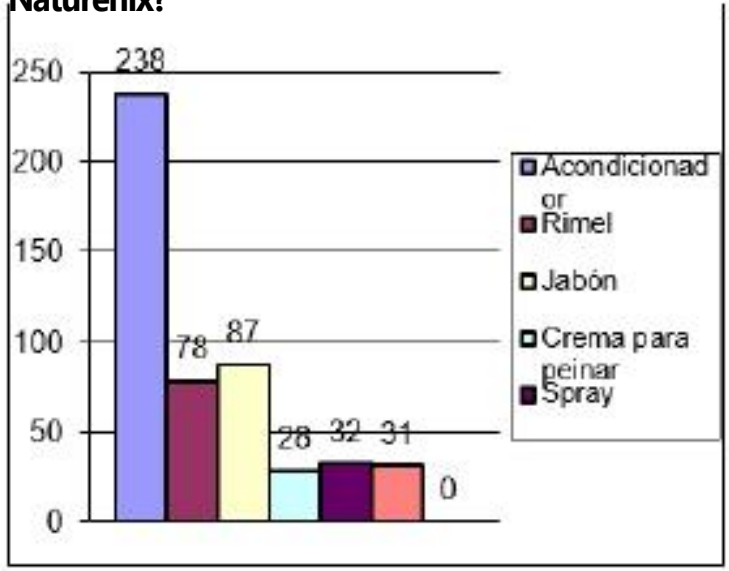

En cuanto a la recompra del producto la encuesta arrojo que la gran mayoría de compra Shampoo al mes. Del $45 \%$ de los encuestados que manifestaron utilizar Naturenix mencionaron que lo comparte con otro familiar.

\section{9. ¿Con Que frecuencia compras Shampoo?}

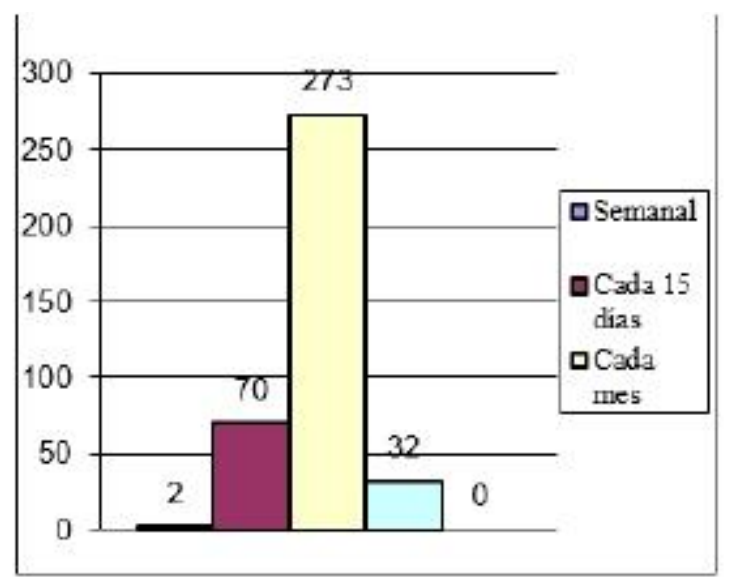

10. ¿Alguien más utiliza Shampoo Naturenix en su hogar?
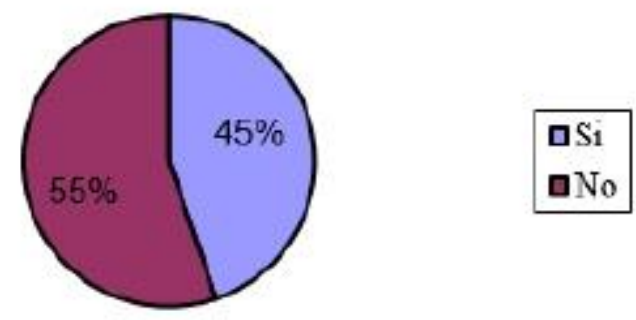

Uno de los datos más interesantes obtenidos en la encuesta es que quienes compran el Shampoo Naturenix lo comparten con algún familiar. No importa el sexo o la edad. La necesidad de detener la caída del cabello o fortalecerlo se manifiesta sin importar el sexo o la edad. También se debe considerar que el Shampoo Naturenix no recomienda su uso diario, lo cual facilita a todos los integrantes de un hogar hacer uso de este producto ocasionalmente. 


\section{Prueba de hipótesis}

1. Hipótesis: Al menos $80 \%$ de las personas encuestadas han utilizado en alguna ocasión un Shampoo para combatir la caída del cabello.

Pregunta para contrastar: ¿Has utilizado algunas ves Shampoo para la caída del cabello o para prevenir la caída del cabello?

\begin{tabular}{|c|c|c|}
\hline Opciones & Frecuencias & $\%$ \\
\hline Si & 264 & $68.75 \%$ \\
\hline No & 120 & $31.25 \%$ \\
\hline Total: & 384 & $100 \%$ \\
\hline
\end{tabular}

msestraiplea una prueba de una proporción con una

$$
S p=\sqrt{\frac{P(1-P)}{n-1}}=\sqrt{\frac{0.80(1-0.80)}{384-1}}=0.020
$$

(Ver Fórmula 1)

$$
Z=\frac{0.6875-0.80}{0.020}==5.625>1.645
$$

Entonces, se puede decir con un $95 \%$ de confiabilidad que hay diferencia significativa entre la Proporción

Observada y la Proporción según la Hipótesis Nula, por lo que se rechaza la Hipótesis Nula. El número de personas encuestadas que han utilizado el Shampoo para evitar la caída de cabello es inferior al $80 \%$ esperado.
2. Hipótesis: El $40 \%$ de los encuestados conoce el Shampoo Naturenix.

Pregunta para contrastar: ¿Conoce el Shampoo Naturenix?

\begin{tabular}{|c|c|c|}
\hline Opciones & Frecuencias & $\%$ \\
\hline Si & 350 & $91.14 \%$ \\
\hline No & 34 & $8.86 \%$ \\
\hline Total: & 384 & $100 \%$ \\
\hline
\end{tabular}

Ste explea una prueba de una proporción con una muestra:

$$
\begin{gathered}
p=\sqrt{\frac{0.40(1-0.40)}{384-1}}=0.025 \\
S Z=\frac{0.9114-0.40}{0.025}=20.456>1.64
\end{gathered}
$$

Entonces, se puede decir con un 95\% de confiabilidad que hay diferencia significativa entre la Proporción Observada y la Proporción según la Hipótesis Nula, por lo que se rechaza la Hipótesis Nula. En este caso, el número de personas encuestadas que han utilizado el Shampoo Naturenix es muy superior al $40 \%$ esperado.

Fórmula 1

$\mathrm{Z}=\frac{\text { Proporción Observada }- \text { Proporción según la Hipótesis Nula }}{\mathrm{Sp}}$ 
3. Hipótesis: El $90 \%$ consideran la calidad del Shampoo Naturenix como buena.

Pregunta para contrastar: ¿Cómo considera la calidad del producto?

\begin{tabular}{|c|c|c|}
\hline Opciones & $\begin{array}{c}\text { Frecuencia } \\
\text { s }\end{array}$ & $\%$ \\
\hline $\begin{array}{c}\text { Muy } \\
\text { buena }\end{array}$ & 118 & $\begin{array}{c}30.73 \\
\%\end{array}$ \\
\hline Buena & 234 & $60.9 \%$ \\
\hline Regular & 32 & $8.3 \%$ \\
\hline Mala & 0 & 0 \\
\hline Muy mala & 0 & 0 \\
\hline Total: & 384 & $100 \%$ \\
\hline
\end{tabular}

Ste eelpoplea una prueba de una proporción con una muestra:

$$
\begin{gathered}
S p=\sqrt{\frac{0.90(1-0.90)}{384-1}}=0.015 \\
Z=\frac{0.9163-0.90}{0.015}=1.086>1.645
\end{gathered}
$$

Entonces, se puede decir con un $95 \%$ de confiabilidad que no hay diferencia significativa entre la Proporción Observada y la Proporción según la Hipótesis Nula, por lo que se acepta la Hipótesis Nula. En este caso, el porcentaje de respuestas que consideran al Shampoo Naturenix entre Muy Bueno (30.73\%) y Bueno (60.9) equivalen al $91.63 \%$ el cual al evaluar la prueba de hipótesis no presenta diferencia significativa con el $90 \%$ esperado.

\section{Conclusiones}

La Compañía Jayan creada por jóvenes emprendedores universitarios, encontró un hueco en el mercado. Un Shampoo que evita la caída del cabello y además lo fortalece, pero lo más importante es que se trata de un producto natural.
Sus ventas son consideradas "adecuadas" pero la necesidad de conocer mejor su mercado les obligo a buscar apoyo para hacer un análisis interno de la empresa, posteriormente un análisis del mercado. Lo anterior para conocer la situación real de su producto, sus alcances y posicionamiento. Pero todavía más importante, ver la posibilidad de ampliar el portafolios de productos de la empresa.

La necesidad de obtener información hizo necesario llevar a cabo una investigación de mercado para conocer mejor su posición y evaluar tres puntos principales:

1. La cantidad de personas con problemas de caída de cabello o con la necesidad de fortalecerlo, y que han utilizado algún producto para ese propósito.

2. El grado de conocimiento de producto.

3. La percepción de la calidad (confiabilidad) de los atributos del producto).

Los resultados fueron contundentes:

La necesidad de evitar la caída de cabello y fortalecerlo existe, pero no utilizan productos para satisfacer dicha necesidad, o no aceptan que lo hacen.

No es suficiente la cantidad de personas que conocen el producto, hay que utilizar mejores estrategias de comunicación y distribución del producto.

La percepción de la calidad del producto es alta, lo cual es un beneficio tangible que debe aprovechar la empresa.

De acuerdo a los resultados antes mencionados, la Compañía Jayan tiene posibilidades para aumentar sus ventas, desarrollar estrategias de comunicación integral de marketing para mejorar el conocimiento de su producto y aumentar su posicionamiento aprovechando las ventajas que le otorga su imagen de producto de buena calidad. 


\section{Bibliografía}

1. Baena Gracia, Verónica (2014) Fundamentos de Marketing: entorno, consumidor, estrategia e investigación comercial. Primera Edición. Editorial UOC SL. Madrid, España.

2. Best, Roger, J. (2007) Marketing Estratégico. Cuarta Edición. Editorial Pearson. España

3. Díaz, P., C.A. y J. Cavazos A. (2014) Investigación de Mercados para pequeñas y medianas empresas. Primera Edición. Editorial Universitaria (UAG). Guadalajara, Jalisco.

4. Fernández Valiñas, Ricardo (2015)

Segmentación de Mercados. Tercera Edición. Editorial McGraw Hill. México, D.F.

5. Hoyer, W.D., D.J. Macinnis y R. Pieters (2015) Comportamiento del Consumidor. Séptima Edición. Editorial Cengage. México, D.F.

6. Mas Ruíz, Francisco José (2010). Temas de Investigación Comercial. Quinta Edición. Editorial Club Universitario. España.

7. McDaniel, C. y R. Gates (2016) Investigación de Mercados. Décima Edición. Editorial Cengage. México, D.F.

8. Metzberg, Michael y Víctor Donaire (2007) Gerencia estratégica de mercadeo. Primera Edición Ed. Thompson. México, D.F.

9. Plazas Rojas, Flavio (2011) Investigación de mercados: un enfoque gerencial. Ediciones de la $\mathrm{U}$. Colombia.

10. Rivas, J.A. y I. Grande E. (2012) Comportamiento del Consumidor: Decisiones y estrategias de marketing. Sexta Edición. ESIC Editores. México, D.F.

11. Schiffman, Leon G. y L. Lazar K. (2010) Comportamiento del Consumidor. Décima Edición. Editorial Pearson. México, D.F.

12. Valderrey Sanz, Pablo (2010) Investigación Comercial: Métodos y aplicaciones. Editorial Starbook. Madrid, España. 